Special issue of the International Conference on Computational and Experimental Science and Engineering (ICCESEN 2014)

\title{
A Zero -Index Metamaterial Single Layer Superstrate for Patch Antenna Gain Enhancement
}

\author{
C.M. Saleh*, D. Bensafieddine, E.M. LaAmari and M. Bouzouad \\ Telecommunications, Signal and Systems Laboratory, BP 37G, Route de Ghardaïa, 03000, Laghouat, Algeria
}

The objective of this paper is to use a zero refractive index $n$ metamaterial as single layer superstrate suspended above a microstrip patch antenna (MPA), operating at $43 \mathrm{GHz}$, for the gain enhancement. The single metamaterial layer superstrate is composed of a periodic arrangement of Jerusalem cross unit-cells, and behaves as an homogeneous medium characterized by a refractive index close to zero. This metamaterial low index property allows gathering radiated waves from the antenna and collimates them towards the superstrate normal direction. The proposed design improves the antenna gain by $5.1 \mathrm{~dB}$.

DOI: 10.12693/APhysPolA.128.B-307

PACS: 78.67.Pt, 84.40.Ba

\section{Introduction}

In antenna engineering domain, researchers have to resolve many fundamental challenges [1]. Antennas have to conjugate at the same time many characteristics which are often conflicting. The use of structured artificial materials, can offer interesting solutions in this domain [2-5]. In this paper, we us an array of Jerusalem cross type metamaterial unit cells with an optimum periodicity to design a zero refractive index metamaterial (ZIM). The ZIM material is employed as a single layer superstrate (ZIM-SLS) above a microstrip patch antenna (MPA). This metamaterial superstrate allows gathering radiated waves from the antenna and collimates them towards the superstrate normal direction and can be effectively used for the antenna gain enhancement.

\section{Metamaterial unit cell characterization}

The Jerusalem cross elementary unit cell is sketched in Fig. 1a. We use a dielectric substrate with permittivity $\varepsilon_{r}=2.2$, thickness $d=0.8 \mathrm{~mm}$ and metallization thickness $t=0.035 \mathrm{~mm}$ [3]. The conducting strip had width $w$, metallization thickness $t$, substrate permittivity $\varepsilon_{r}$ and thickness $d$ (Fig. 1b). Since we have fixed some of them $\left(t, \varepsilon_{r}\right.$ and $\left.d\right)$, we use $w$ as design parameter to tune the metamaterial unit cell behavior. A parametric study is used to obtain the dimensions of the metamaterial unit cell. The constitutive parameters $\left(\varepsilon_{\text {eff }}\right.$ and $\left.\mu_{\text {eff }}\right)$ and the refractive index of the metamaterial, shown in Fig. 2, are obtained with the Fresnel inversion method using the $S$ parameters of the unit cell [4]. The $S$ parameters are obtained by a finite difference method based on a commercial simulation code. It can be seen from Fig. 2b the Jerusalem cross based metamaterial exhibits a plasmonic-type permittivity frequency function. For frequencies larger than the plasma frequency the relative permittivity stays between 0 and 1 .

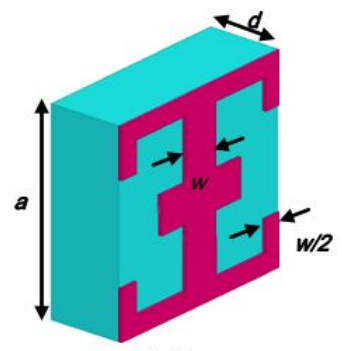

(a)

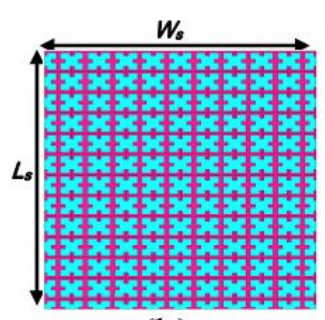

(b)

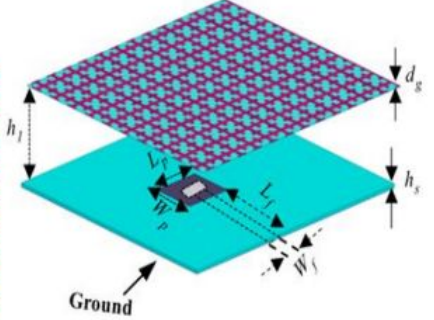

(c)

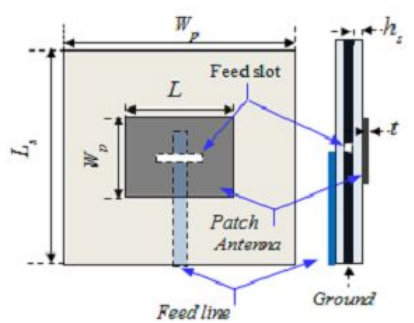

(d)

Fig. 1. The proposed antenna: (a) unit- cell; (b) bottom view of ZIM superstrate layer; (c) perspective view; (d) Antenna slot feeding details: $h=0.8, W=0.2, t=0.035, a=2, L_{s}=25, W_{s}=20, h_{s}=0.05, d_{g}=1.6, L_{p}=0.257$, $W_{p}=0.184, h_{1}$ and $h_{2}$ in [mm]. 
The metamaterial presents a plasma frequency equal or a little less than $40 \mathrm{GHz}$ and a very low real part (close to zero), in the operating frequency range (40-45 $\mathrm{GHz}$ ), which corresponds to the desired profile. Also, the effective permeability presents a profile close to unity. It can be seen that the constitutive parameters $\left(\varepsilon_{\text {eff }}\right.$ and $\left.\mu_{\text {eff }}\right)$ have positive and close to zero imaginary parts which attests to the low-loss nature of the effective medium. From Fig. 2a, it can be seen that, in the operating frequency range $40-45 \mathrm{GHz}$, the refractive index presents a flat profile close to zero corresponding to the desired ultra refraction $(0<n<1)$ low dispersive behavior for the ZIM effective medium [5]. These results are obtained for conductor strip width $w=0.2 \mathrm{~mm}$. The MSP antenna operating frequency is $43 \mathrm{GHz}$, consequently the corresponding wavelength in free space is $7.2 \mathrm{~mm}$. Accordingly, the period $a$ and hence the greatest dimension of the metamaterial unit cell must be much lower than the operating wavelength, to respect the homogenization criterion [6]. So it's fixed at $a=2 \mathrm{~mm}$ (lower than $\lambda / 3 \approx 2.4 \mathrm{~mm}$ ). The metamaterial refractive index is shown in Fig. 2b. The real part is clearly very close to zero over the frequency range 40-45 GHz.
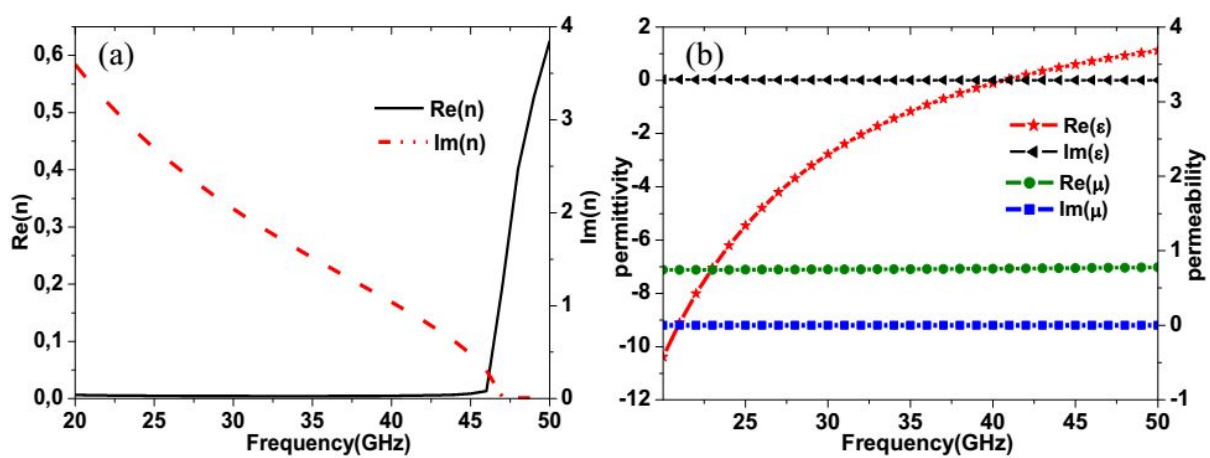

Fig. 2. (a) Refractive index of the Jerusalem cross type metamaterial effective medium, (b) Effective permittivity and permeability of the Jerusalem cross type metamaterial effective medium.
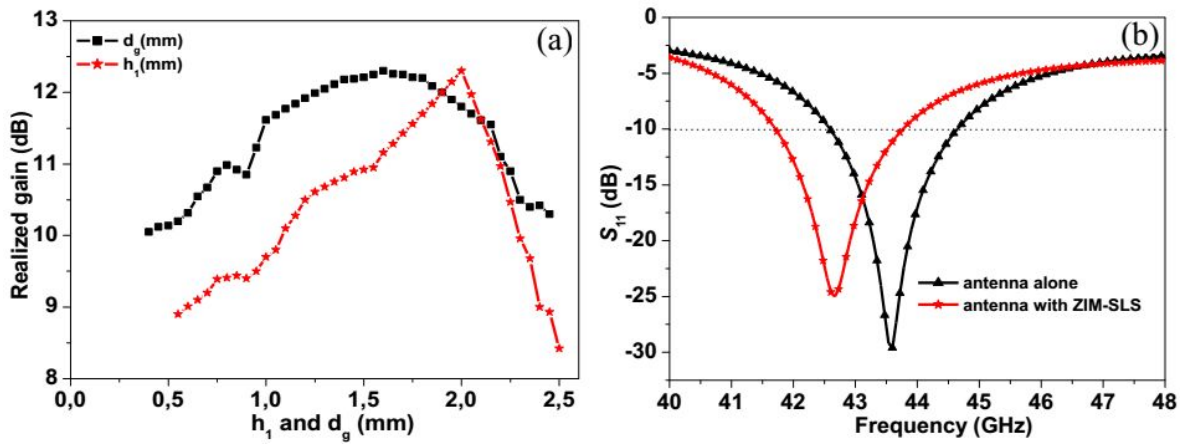

Fig. 3. (a) Realized gain of the patch antenna versus the ZIM-SLS spacing $h_{1}$ and thickness $d_{g}$, (b) Patch antenna with and without ZIM superstrate reflection coefficient.

\section{High gain antenna configuration}

Our designed high gain antenna is depicted in Fig. 1c and $\mathrm{d}$. The structure is formed by a slot feed microstrip patch antenna (MPA) on which the ZIM-SLS is superposed. The patch antenna with dimensions $W_{p} \times L_{p}=$ $(0.184 \times 0.257) \mathrm{mm}^{2}$, operating at $43.5 \mathrm{GHz}$. The dielec- tric substrate with permittivity constant $\varepsilon_{r}=2.2$ and a thickness $d=0.8 \mathrm{~mm}$. The basic building block of the metamaterial superstrate is the ZIM unit-cell as displayed in Fig. 1a with geometrical parameters $a, w$, and $t$. The unit-cells are arranged in an array with periodicity of $12 \times 10$, to design a layer of the ZIM superstrate, as shown in Fig. 1b. The metamaterial superstrate covers 
the patch antenna and it functions like a lens to improve the antenna gain and directivity. The spacing from the radiator to the bottom of the ZIM superstrate is $h_{1}$.
The antenna ground plane and the ZIM superstrate have dimensions of $\left(L_{S} \times W_{S}=25 \times 20\right) \mathrm{mm}^{2}$.
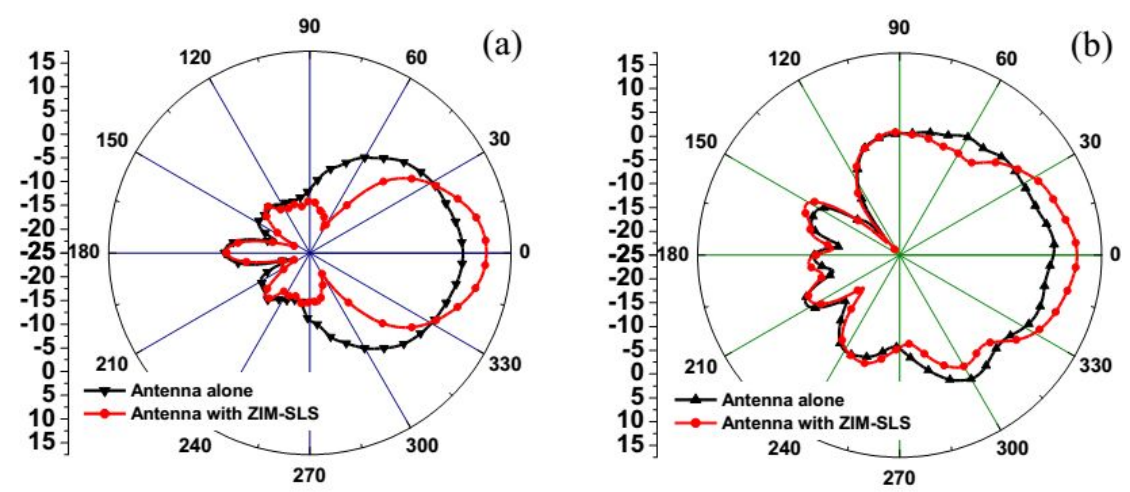

Fig. 4. Radiation pattern of the patch antenna with and without ZIM superstrate: (a) $E$-plane $\left(\varphi=90^{\circ}\right)$, (b) $H$-plane $\left(\varphi=0^{\circ}\right)$

\section{Results and discussion}

From Fig. 3a, it can be seen that $d_{g}$ the thickness of the superstrate layers, $h_{1}$ the spacing between the patch antenna and the superstrate layer are key parameters in the antenna gain improvement. A parametric study shows that there are optimal values for $d_{g}$ and $h_{1}$ for which the antenna gain is maximized. These are $d_{g}=1.6 \mathrm{~mm}$, and $h_{1}=2 \mathrm{~mm}$. It is observed from Fig. $3 \mathrm{~b}$ that the resonance of the MPA is slightly shifted to lower frequency region by about $2 \%$ when loaded with the ZIM superstrate. The maximum realized gain of the proposed antenna is found to be $12.3 \mathrm{~dB}$ with a ZIM-SLS. If compared to the MPA on the same Rogers RT substrate, our proposed antenna design with a ZIM-SLS exhibits a gain enhancement of about $70 \%$ (Table). The antenna

TABLE

Half-power beam width $\theta_{-3 \mathrm{~dB}}[\mathrm{deg}]$ and realized gain of the patch antenna with and without ZIM superstrate.

\begin{tabular}{|c|c|c|c|c|}
\hline & \multicolumn{2}{|c|}{$\theta_{-3 \mathrm{~dB}}[\mathrm{deg}]$} & \multirow{2}{*}{$\begin{array}{c}\text { realized gain } \\
{[\mathrm{dB}]}\end{array}$} & \multirow{2}{*}{$\begin{array}{c}\text { band width } \\
{[\%]} \\
\end{array}$} \\
\hline & $E$-plane & $H$-plane & & \\
\hline antenna alone & 66.5 & 80.1 & 7.2 & 4.6 \\
\hline $\begin{array}{c}\text { antenna } \\
\text { with ZIM-SLS }\end{array}$ & 33.6 & 44.8 & 12.3 & 4.7 \\
\hline
\end{tabular}

radiation patterns are illustrated in Fig. 4. It is clear from the plot that loading a microstrip antenna with the ZIM based superstrate, focuses the radiation beam width and thereby enhances the antenna directivity. We observe a half power beam width reduction of about $50 \%$ with a ZIM-SLS, in both the H-plan and E-plane as shown in Table. We note that the ZIM-SLS reduces the radiation pattern half power beam width by about $50^{\circ}$ and shifts the antenna operating frequency by about $2 \%$, while it doesn't affect consequently the band width.

\section{Conclusion}

We have proposed a high gain antenna based on a zero index metamaterial superstrate. A gain enhancement up to $5.1 \mathrm{~dB}$, and about $50 \%$ half power beam width reduction have been obtained for the proposed design compared to a microstrip patch antenna alone. We observe also a shift by about $2 \%$ of the MPA operating frequency.

\section{References}

[1] B.A. Munk, Metamaterial: Critique and Alternatives, Wiley, New York 2009.

[2] Dalin Jin, Bing Li, Jingsong Hong, in: Int. Conf. Microwave Millimeter Wave Techn. (ICMMT) 2012 3, 1 (2012).

[3] M. Bouzouad, Ph.D. Thesis, ENP Alger, Alger 2007.

[4] D.R. Smith, Phys. Rev. E 71, 036617 (2005).

[5] N. Engheta, R.W. Ziolkowski, Metamaterials physics and engineering explorations, Wiley, 2006.

[6] S.A. Tretyakov, I.S. Nefedov, C.R. Simovski, S.I. Maslovski, Electromagnetics 22, 177 (2002). 